\title{
Universidad Internacional para la Salud de los Pueblos 2011 - 2018. Los Cursos Uisp en El Salvador, una experiencia transformadora
}

\author{
International People's Health University 2011 -2018. The IPHU \\ Courses in El Salvador, a transformative experience
}

Maria Elizabeth Hamlin Zúniga1

DOI: 10.1590/0103-11042020S115

RESUMEN El Ministerio de Salud de El Salvador (Minsal) y el Movimiento para la Salud de los Pueblos (MSP) implementaron siete cursos dirigidos a personal de salud y líderes comunitarios comprometidos, o con deseos de serlo, en el ejercicio del derecho humano a la salud por la población, con apoyo de la Universidad Internacional para la Salud de los Pueblos (Uisp) durante el periodo 2011-2018 (gobierno del Frente Farabundo Marti para la Liberación Nacional - FMLN). Se formaron ocho generaciones incluyendo más de 300 personas, entre personal del Minsal, otras instituciones, líderes del Foro Nacional de Salud y líderes comunitarios latinoamericanos. El artículo comparte objetivos, metodología, proceso de organización e implementación, y resultados de esta experiencia. Se analiza el impacto del proceso de formación de equipos de salud y referentes de movimientos sociales, ejecutando un acuerdo-alianza entre Estado y Sociedad Civil. También se comparte el impacto en el fortalecimiento del sistema de salud salvadoreño y unos registros de las vivencias y sentipensares de algunos participantes. El articulo permite comprender las oportunidades que genera este tipo de alianza así como las dificultades encontradas y como se las superaron.

PALABRAS CLAVES Reforma de salud. Profesionales de la salud. Movimiento social.

\begin{abstract}
The Ministry of Health of El Salvador (Minsal) and the People's Health Movement (PHM) implemented seven courses for health personnel and community leaders committed, or with the desire to be, in the exercise of the human right to health, with support from the International People's Health University (IPHU) during the period 2011 - 2018 (government of the Farabundo Marti Liberation Front - FMLN). Eight generations were formed including more than 300 people, comprising Minsal staff, other institutions, leaders of the National Health Forum and Latin American community leaders. The article shares objectives, methodology, process of organization and implementation, and results of this experience. The impact of the process of formation of health teams and activists of social movements is analyzed, achieving an agreementalliance between State and Civil Society. The impact on the strengthening of the Salvadoran health system and some records of the experiences and reflections (sentipensares) of some participants are also shared. The article allows us to understand the opportunities generated by this type of alliance as well as the difficulties encountered and how they were overcome.
\end{abstract}

1 Movimiento para la Salud de los Pueblos - Managua, Nicaragua.

maria.hamlin.zuniga@gmail. com
KEYWORDS Health care reform. Health professionals. Social movement. 


\section{Introducción: nacimiento del MSP y la Uisp}

En 2005 el Movimiento para la Salud de los Pueblos (MSP) organizó su segunda Asamblea Mundial en Cuenca, Ecuador. Previo a la Asamblea, se implementó el primer curso de la Universidad Internacional para la Salud de los Pueblos (Uisp), con una duración de diez días. En éste, participaron estudiantes y docentes de Centroamérica, como Eduardo Espinoza, Vice Ministro de Políticas de Salud de El Salvador, Margarita Posada, actualmente Coordinadora del Foro Nacional de Salud de El Salvador, y María Hamlin Zúniga de Nicaragua, en ese momento, coordinadora del Consejo Internacional de Salud de los Pueblos. Entre los acuerdos de la segunda Asamblea estuvo el compromiso de promover la Uisp en todo el mundo, retomando el modelo del primer curso en Cuenca, Ecuador.

Después de la segunda Asamblea, el MSP realizó cursos Uisp en Latinoamérica, África, Asia, Australia, Medio Oriente, Europa y Norte América. Algunos fueron de diez días completos, como el de Cuenca, y otros de menor duración, como sesiones educativas de uno o dos días o seminarios de varios días.

En los cursos Uisp, emblemáticos de MSP, participaron jóvenes de diferentes países, por lo regular conducido en inglés y con docentes integrados en la conducción del MSP global o en expresiones por la salud propias de la región donde se desarrollaba el curso. Estos docentes contaban con una amplia formación y reconocimiento académicos, igual que un claro compromiso con las luchas por el derecho a la salud.

\section{Las bases conceptuales para los cursos Uisp en El Salvador}

El objetivo de la Uisp es contribuir a la 'salud para todos y todas' con el fortalecimiento del MSP como una red de organizaciones y activistas en todo el mundo. Para ello asume el compromiso de desarrollar capacidades en activistas que trabajan por un mundo saludable, justo, armonioso y sostenible.

En correspondencia con este objetivo, la Uisp en El Salvador organizó cursos intensivos de 7 a 10 días, con 30 a 40 jóvenes activistas y docentes locales e internacionales, trabajando juntos en temas como Atención Primaria de Salud (APS), promoción de la salud, economía política de salud, reformas del sector salud, salud y comercio, género y salud, determinación social de la salud, concientización, investigación participativa y cómo trabajar con las comunidades.

Actualmente, además del enfoque hegemónico, hay diferentes enfoques sobre la salud a nivel mundial, uno que habla de Determinantes Sociales de la Salud y otro de Determinación Social de la Salud. El Ministerio de Salud de El Salvador (Minsal), ha hecho una apuesta por poner en práctica el enfoque de determinación social de la salud.

\section{Reforma de Salud en El Salvador}

Las vivencias de las Asambleas Mundiales de Salud de los Pueblos y de la Uisp generaron respaldo en Centroamérica a los planteamientos de estas; entre otras a promover la APS y continuar la lucha con énfasis en la determinación social de la salud. Activistas centroamericanxs y particularmente salvadoreñxs fueron impactadxs por la experiencia, lo que incidió en sus conocimientos, investigaciones y activismo contra la privatización de la salud a nivel comunitario y nacional.

En 2009, la izquierda salvadoreña ganó las elecciones presidenciales. Previamente, había preparado su plataforma electoral, identificando las necesidades de la población. La propuesta integraba elementos ampliamente discutidos en las Asambleas Mundiales del MSP y en los cursos Uisp. El lema de la campaña electoral fue 'Nace la esperanza, 
viene el cambio', lo que incidió en el diseño de la Política Nacional de Salud que debía llevar adelante el nuevo gobierno.

La Política Nacional de Salud de El Salvador 'Construyendo la Esperanza'1 definió como su objetivo 'Garantizar el derecho humano a la salud a toda la población', concibiendo a los recursos humanos en salud como la piedra angular de su Reforma de Salud. Priorizó la APS como la estrategia a seguir por el sistema de salud tanto con la población como en todos los establecimientos de salud (especialmente las Unidades Comunitarias de Salud Familiar y la red hospitalaria). La política de salud desarrolló también el abordaje de Determinación Social de la Salud y aplicó la epidemiología crítica como herramienta fundamental de trabajo. Este abordaje retomó los aportes epistemológicos y el amplio debate que se venía dando desde la década de los años 70 en el seno de la Asociación Latinoamericana de Medicina Social (Alames) sobre las limitaciones del enfoque de riesgo y su versión más reciente de Determinantes Sociales de la Salud. Al centro del debate se discutía la necesidad de superar el abordaje fraccionado de la realidad al que inducían 'los Determinantes Sociales de la Salud', que a su vez conducían a ocultar que el problema fundamental lo constituía el modelo hegemónico de desarrollo, centrado en el crecimiento económico infinito en un mundo con recursos naturales finitos. Este modelo en su conjunto (y no parcializado en determinantes) era quien condicionaba la depredación, el hiperconsumismo, las migraciones, el extractivismo, la crisis ambiental y el calentamiento, todo ello con dimensiones globales. De esta manera el abordaje de 'Determinantes Sociales de la Salud' como versión del enfoque de riesgo se convertía en un obstáculo para avanzar hacia un nuevo modelo de desarrollo en armonía con el entorno a través de una relación racional entre la humanidad y la naturaleza.

En 2009, durante una discusión en la Universidad de El Salvador sobre la nueva política de la Reforma en Salud, se identificó la necesidad de dar mayor impulso a la APS en el país y realizar cursos para concientizar al personal de salud y al movimiento social en gestación.

El Dr. David Sanders, uno de los fundadores del MSP, en una visita de trabajo a El Salvador en marzo de 2011, planteó la idea de la formación de activistas dentro del marco de la Reforma en Salud y recomendó la realización de cursos Uisp en el país como una oportunidad única de trabajar directamente con un ministerio de salud en un proyecto emblemático del MSP.

Con la toma del gobierno por el FMLN, se abrieron las posibilidades de hacer cursos permanentes en El Salvador, lo que era necesario aprovechar. La experiencia salvadoreña es la más intensa en América Latina en términos de una reforma en salud que privilegia la atención primaria y comunitaria por encima del enfoque curativo, al tiempo que incluye como eje fundamental la participación social, representada actualmente por el Foro Nacional de Salud (FNS). El gran objetivo del FNS es la toma de decisiones consensuados para la construcción del nuevo Sistema Nacional de Salud basado en el ejercicio pleno del derecho a la salud por la población, la universalidad, la inclusión, la calidad y el protagonismo ciudadano. El Foro tiene tres objetivos estratégicos:

1. Participación social significante (políticas de salud, toma de decisiones, evaluación)

2. Desarrollo organizativo territorial (por comunidades, municipios, departamentos y nacional) y desarrollo organizativo sectorial: derechos en salud sexual y salud reproductiva, medicamentos, cambio climático, mujeres, jóvenes, etc, y

3. Incidencia para empoderar a la población sobre el conocimiento y los alcances de su derecho a la salud, a fin de reivindicar ante instancias gubernamentales y no gubernamentales la garantía de este derecho y la satisfacción de las necesidades derivadas del mismo. 
Poco a poco, los contenidos de la Política Nacional de Salud 'Construyendo la Esperanza' y la necesidad de desarrollar los recursos humanos como piedra angular del proceso de reforma, encontraron un elemento renovador y catalizador en los docentes y estudiantes que habían participado en diferentes actividades del MSP incluyendo la Uisp desde su origen.

\section{Ocho generaciones de cursos Uisp en El Salvador}

En septiembre de 2011 se realiza el Tercer Congreso del Movimiento Salvadoreño por la Salud Dr. Salvador Allende y el Primer Congreso de la Asociación Latinoamericana de Medicina Social - Alames-El Salvador denominado 'Dra. María Isabel Rodríguez'. En este marco se solicitó y acordó la adhesión del Movimiento Salvador Allende al MSP.

Esta incorporación al MSP vino a consolidar la oportunidad histórica de organizar en El Salvador el primer curso de la Uisp dirigido a la formación del liderazgo y activistas por el derecho a la salud, particularmente los miembros del Movimiento Por la Salud Dr. Salvador Allende que laboraban como personal de salud y podían catalizar el sistema de salud, sumándose igualmente a los cursos Uisp activistas y liderazgo del Foro Nacional de Salud y egresados de la Escuela Latinoamericana de Medicina en Cuba (Elam). Los siguientes pasos incluyeron el trabajo de diseño, la búsqueda de financiamiento y la identificación de personas, especialmente aquellas que desde los años 70 venían contribuyendo al pensamiento crítico y progresista en América Latina, incluyendo profesionales de Alames y el Movimiento para la Salud de los Pueblos América Latina (MSPLA). En esta oportunidad, se buscó eliminar la barrera lingüística que estuvo presente en el curso Uisp de Cuenca y en otros, por lo que se aprovecharon recursos latinoamericanos o de habla española. El primero curso Uisp, que en El Salvador se denominó 'Curso de políticas de salud para la promoción y abogacía del derecho humano a la salud en el marco del proceso de la Reforma de Salud de El Salvador', inició en 2011.

Poco a poco, y como resultado de la cualificación derivada de la presencia del liderazgo del FNS, la participación del liderazgo en el curso se fue incrementando.

A la fecha, se han formado ocho generaciones abarcando ya a más de 300 personas, entre profesionales de la salud y otras disciplinas, y el liderazgo del FNS. Estas personas asumen el compromiso de contribuir a la profundización de la Reforma de Salud en El Salvador, que es el fruto de un intenso debate en el seno del Movimiento Social, instancias gremiales y académicas, comités de base del partido FMLN, población en general y solidaridad internacional que se resumen en el documento de política 'Construyendo la Esperanza' ${ }^{\prime, 2}$. El planteamiento básico de la reforma rechaza la mercantilización de la salud y la concibe como un derecho humano fundamental. Se ha dado oportunidad de participar en los cursos a personas de otros países de la región, en particular Guatemala, Honduras y Nicaragua, además de una persona de Bolivia, una de Ecuador y dos del País Vasco.

El proceso inició con el diseño de un proyecto de gestión para la obtención de fondos. Se conformó el equipo planificador académico y de logística, que elaboró la programación del curso, identificó y convocó a lxs docentes más idóneos nacionales e internacionales; también se integró el equipo de facilitación constituido con lxs participantes más destacadxs de las generaciones anteriores. Luego, se realizó la convocatoria que fue publicada en el sitio web del Minsal. Desde entonces, el proceso de selección y reclutamiento es el mismo: la persona llena el formulario y presenta una propuesta de proyecto, que puede ser una investigación o una actividad de promoción de la salud. A ésta se debe adjuntar el permiso de su jefe y el compromiso con las reglas internas de la Uisp. Posteriormente, se desarrolla un proceso de selección donde el equipo coordinador del curso revisa las solicitudes y las califica. Se toma 
en cuenta la edad, género, profesión, ubicación dentro del sistema, experiencia de activismo, se califica su proyecto y se recomienda si se incluye o no. La coordinación busca la equidad de género en la selección, prioriza a personas menores de 40 años con distribución equitativa en las cinco regiones de salud del país, instituciones formadoras, organismos no gubernamentales, asociaciones $\mathrm{u}$ organizaciones profesionales, de diferentes disciplinas y niveles de atención. Posteriormente, se notifica a las personas seleccionadas y se gestiona una carta de permiso para ausentarse de su puesto de trabajo. En el caso del FNS, la coordinación técnica, propone a sus participantes siguiendo criterios similares tomando en cuenta la representatividad territorial. En cuanto a la edad, se permite que el FNS tenga un mayor rango. Al final del curso, se realiza la evaluación integral en línea y los resultados se discuten principalmente con el equipo de facilitación.

El propósito de este curso en El Salvador ha sido el de generar capacidades técnicas, conductuales y políticas para un abordaje integral de la salud y sus iniquidades, que considere la determinación social como el mejor enfoque para abordarla y tomar control sobre la misma por las comunidades organizadas. El curso se realiza con el acompañamiento académico de la Uisp y del MSPLA y es coordinado por el Vice Ministerio de Políticas de Salud y un equipo técnico.

El curso dura diez días continuos, a tiempo completo y con largas jornadas de trabajo. Ello implica que lxs 35 participantes, lxs facilitadores y lxs profesores nacionales e internacionales, expertxs en salud pública, se concentran por ese período en un lugar fuera de San Salvador. Se forman grupos de trabajo permanente para discusiones a profundidad y para elaborar proyectos, y grupos ad hoc para la elaboración de la memoria, el registro fotográfico y el desarrollo de las actividades culturales. También, se integran grupos de no más de diez personas y con diversas disciplinas para visitas de campo, que tienen como objetivo observar y documentar en el terreno casos concretos del abordaje de la Determinación Social de la Salud por el proceso de reforma salvadoreño, tales como la minería metálica, la enfermedad renal crónica, violencia y salud, contaminación ambiental y actividades del FNS.

Los temas de los cursos Uisp en El Salvador coinciden con los de la currícula global, con la diferencia que éstos se desarrollan en el marco de la Reforma de Salud. Lxs docentes hacen su presentación presencial o virtual, seguida de discusiones en grupos y plenarias. Para las últimas dos generaciones, se ha diseñado una plataforma virtual en el sitio web del Minsal donde se comparten en tiempo real las conferencias y debates con lxs cursillistas.

\section{Caracterización de participantes en cursos Uisp en El Salvador en el período 2011 a 2018}
A lo largo de 7 años, en los cursos Uisp en El Salvador han participado como cursillistas un total de 268 personas. En cada cohorte participaron entre 30 a 40 personas (gráfico 1). 
Gráfico 1. Número de participantes por curso Uisp, El Salvador 2011 - 2018

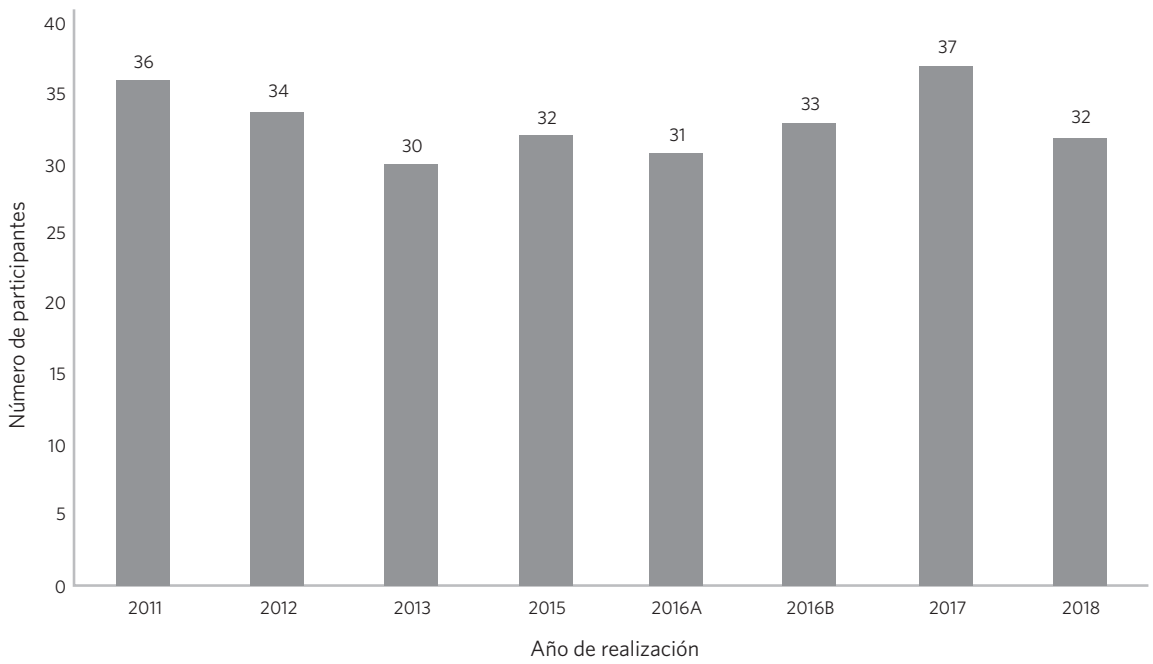

Se procuró una distribución equitativa por género en cada generación (gráfico 2):

Gráfico 2. Distribución de participantes por sexo, cursos Uisp, El Salvador 2011 - 2018

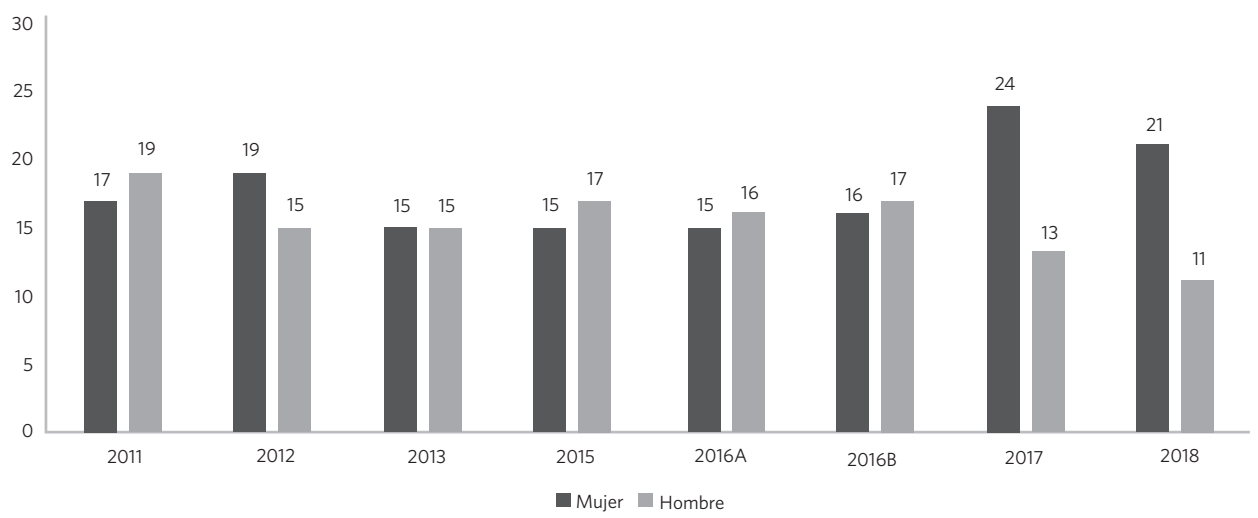

En términos generales, el 54\% (145) fueron mujeres y el $46 \%$ hombres (123), sin embargo, en 2017 hubo mayor participación de mujeres, en su mayoría lideresas del FNS.

Todas las personas que participaron en los cursos eran mayores de edad, los grupos de edad con más representación son el de 35 a 39 años con el $24 \%$ (63), seguido por los grupos de
30 a 34 (54) y de 40 a 44 años (54) con el 20\% en cada grupo. A pesar de ser requisito en los criterios de aplicación al curso ser menor de 40 años, se incluyó a personas que sobrepasan esta edad tomando en cuenta su compromiso y liderazgo desarrollado en espacios de participación social en el FNS y en los espacios de gestión del Sistema Nacional de Salud (gráfico 3). 
Gráfico 3. Distribución de participantes por grupo de edad, cursos Uisp, El Salvador 2011 - 2018

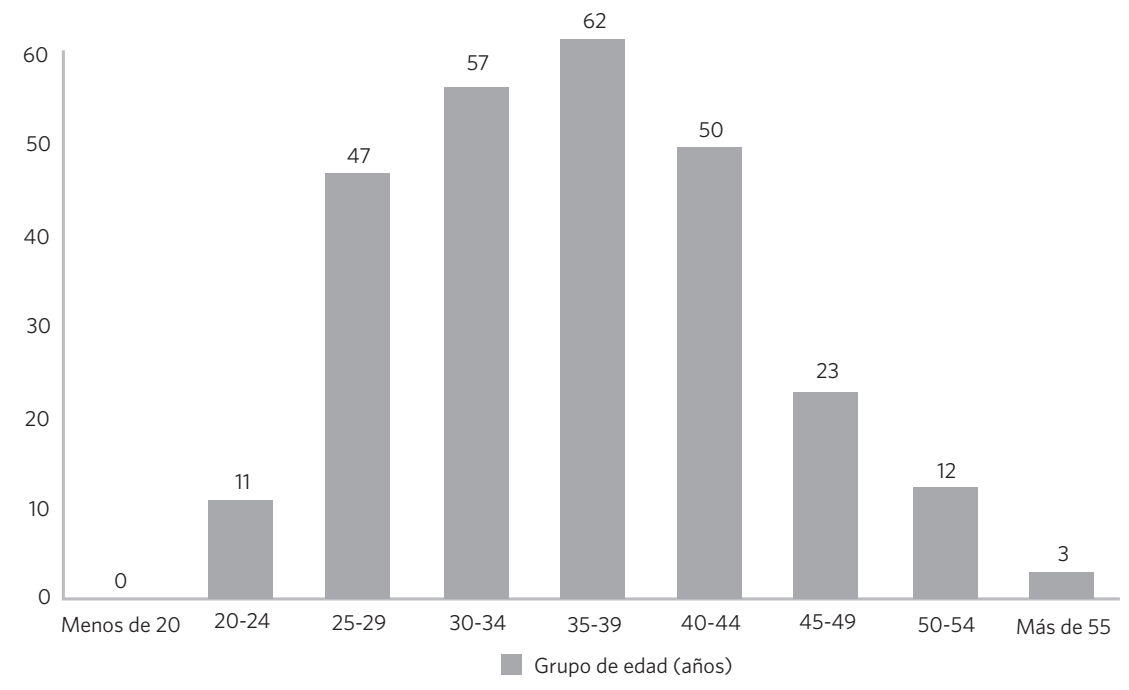

Desde 2016, en la elaboración de un estudio de caso para el proyecto de investigación del MSP, financiado por el International Development Research Center (IDRC) sobre Sociedad Civil y Salud para Todxs (2016) ${ }^{3}$ y en la sistematización de los cursos $(2017)^{4}$ se realizaron entrevistas a profundidad con actores claves, grupos focales, personal de salud y liderazgo del FNS que han participado en algunas de las ocho generaciones del curso Uisp. Se hizo un ejercicio de evaluación cualitativa conocido como 'cambio más significativo' con facilitadores de las diferentes generaciones, así como con estudiantes extranjeros y algunos de los docentes internacionales. El material que sigue es una síntesis de estas actividades.

\section{El cambio más significativo}

El curso Uisp produce un choque intenso que lo sacude a uno. Los contenidos son profundos, muy fuertes y con orientaciones que desvelan 'la realidad real'. Hay un quiebre entre antes y después del curso Uisp. Somos otras personas, ya nadie es igual que antes. (Excursillista).
Algunos profesionales de la salud han opinado que el curso Uisp ayuda a enlazar dos cosas: los conocimientos de la formación profesional universitaria y la política social; es decir, la formación profesional y la militancia. El curso es un enlace entre estas dos formas de pensar, de actuar y de vivir. "Ahora he perdido el miedo de hablar de la salud dentro de mi militancia política", dijo uno de lxs participantes. Consideran que el curso Uisp es valioso desde lo teórico y académico. En ninguna otra parte, se habla de los temas que se desarrollan en el curso Uisp, sobre las diferencias entre la medicina social y colectiva y la práctica biomédica. El curso permite la reflexión y análisis sobre diferentes teorías y visiones de la salud pública.

Comentan activistas del FNS que en el curso Uisp participan personas formadas en áreas no vinculadas a la medicina: "Participar en el curso amplió la visión de que se puede y se debe promover la salud desde distintas áreas disciplinarias". Hay mucha participación activa, así como trabajo en grupos mixtos de personal de salud y liderazgos comunitarios.

Para las y los graduados de la Elam, el curso Uisp es una oportunidad para conocer 
la realidad de El Salvador después de años en un sistema distinto. Ayuda a entender muchos procesos, a esclarecer dudas y es enriquecedora.

El mayor beneficio que me ha traído el curso Uisp es el haber generado hermandad con todas y todos los cursillistas que tenemos un sueño de transformar y construir un mundo mejor. (Excursillista).

El curso Uisp fortalece los lazos de hermandad y comunicación entre hombres y mujeres que tienen el mismo deseo de lucha. Ha aumentado y cualificado las redes de contactos entre activistas y muchos proyectos se han vuelto más fáciles o más rápidos de realizar.

Según militantes del FNS, en el curso hubo algunas diferencias con el personal del Minsal, se manifestó una contradicción entre el discurso y la practica en el territorio; pero sin duda, el curso Uisp es un espacio para generar nuevas relaciones. Después de la participación en el curso, la actitud de algunoxs trabajadorxs de salud cambió con respecto a la imagen que tenían antes.

Ahora puedo ver a Ixs participantes del curso en forma distinta, no por nivel académico sino por ser compañerxs del curso. (Excursillista).

Al principio, había rechazo al liderazgo del FNS por parte del personal de salud. No conocían lo que es el FNS, ni tampoco estas personas sin mucha educación formal, pero con capacidades de liderazgo y apoyo comunitario. El eje de participación social, representada desde 2010 por el FNS, está dentro de los contenidos del curso, aunque es desconocido para la mayoría del personal de salud.

La brecha entre los profesionales y activistas ha sido reducida gracias al curso Uisp.

En el curso, el liderazgo ha entendido porque el médico en las comunidades es prepotente, la razón es porque han sido formados por el sistema hegemónico, por lo que esperamos que se modifique la formación de los profesionales. En el curso, aprendimos mucho del personal de salud, pero ellos conocieron la otra cara del problema y así conocieron el pensar de las comunidades. (Lideresa del FNS).

Para los liderazgos territoriales del FNS, el curso es una motivación que alimenta la autoestima. Participar en una universidad, la Uisp, estudiar con profesionales médicos, con docentes nacionales e internacionales, los satisface. El liderazgo siente las diferencias, pero se dan cuenta de su propio valor y que tienen conocimientos y capacidades diferentes.

La participación del FNS en el curso Uisp, a partir de la segunda generación, ha resultado en el fortalecimiento de su organización, motivación, militancia y compromiso con la Reforma de Salud. El curso da la oportunidad de conocer al liderazgo y su trabajo en la organización comunitaria; al mismo tiempo, permite aclarar su rol en la controversia social y la incidencia política. Según el personal de salud, después de la participación en el curso Uisp, ha cambiado su percepción del FNS.

El curso me hizo bajarme del espacio inexistente en que me tenía el sistema como 'profesional' (colonizado) y me colocó un amarre que me mantiene más cerca de la realidad, esa que critica y hace que duela el dolor de otro. Es un abrir de ojos que sensibiliza y humaniza la persona. (Excursillista).

Hay personal de salud que opina que se tuvo acceso a conocimientos nunca vistos en la formación médica tradicional salvadoreña, sobre una forma distinta de ver la salud, desde la humanización, lo que permite tomar mejores decisiones.

El curso Uisp es una oportunidad para conocer otras personas, otras ideas, de aprender a escuchar a la población. Es una motivación por compartir y llegar a un compromiso personal como agente de cambio. (Excursillista). 
Muchas personas opinan que las visitas de campo fueron una oportunidad que no se tiene en el trabajo cotidiano en los servicios de salud de primer nivel de atención y mucho menos en el nivel hospitalario. Con estas visitas, se pudo conocer de primera mano y por primera vez, la problemática de la minería, los agrotóxicos, la enfermedad renal crónica o la violencia.

El liderazgo del FNS considera que el curso ha ayudado mucho. Ha servido para potenciar la participación de las comunidades. Ahora van a otros municipios para fortalecer la organización, haciendo conciencia de las luchas organizativas. Se tiene más acercamiento con las comunidades; se ha perdido el miedo, ganando más valor. Hay una oportunidad para trabajar nuevos enfoques que ayudan a replantear el enfoque tradicional de la salud. Se ve el aporte de liderazgos en las comunidades, se identifican con estos nuevos enfoques para promover la defensa del derecho a la salud.

El personal docente internacional también fue impactado por el curso:

El curso Uisp representa un importante estímulo para continuar en la lucha por el derecho a la salud y la transformación de la sociedad ya que permite estar en contacto con una experiencia concreta de carácter exitoso. Permite, además, estar en contacto con sus actuales líderes y con potenciales cuadros de dirección del sector salud. El curso Uisp me permite crecer, cada curso implica un aprendizaje importante, por eso trato de estar todo el tiempo, no considero que voy a colaborar con algunas presentaciones o charlas, considero que voy a aprender y a fortalecer mis conocimientos, pero además mis principios y convicciones. (Oscar Feo, Venezuela).

Valoro mucho la concentración que genera la modalidad de trabajo, aunque pienso que puede incrementarse la calidad del mismo si las y los facilitadores desarrollan dinámicas y estrategias metodológicas que hagan que todas y todos participen, de forma que no se baje la guardia con los tiempos, las relatorías e informes de cada grupo. Lo más importante es entender que más allá de la clínica, el trabajo en salud compromete a los mismos derechos humanos y valores más fuertes como la equidad, la justicia, la participación y la movilización del sector en torno a una mejor calidad de vida, de buen vivir y de mejor estar. Abandonar la centralidad androcéntrica y heteronormativa también es importante. La dimensión ambiental y el acercamiento al feminismo y a la perspectiva de género en salud completa muy bien el esfuerzo. (Sara Fernández, Colombia).

Comencé a participar en el Curso Uisp a partir de 2015. Esta ha sido para mí una experiencia formidable e inolvidable en mi vida personal, pues me dio la oportunidad de ver en la práctica el entusiasmo de cohortes de jóvenes profesionales de salud buscando capacitarse para atender las necesidades de salud de la población salvadoreña. Así como enseñé, aprendí mucho de la experiencia de los cursantes y de los demás docentes, de las discusiones colectivas y del intercambio de opiniones. El entusiasmo colectivo se diseminaba a lo largo de todos los cursos. Las amistades generadas en el Uisp perduran en el tiempo y mantengo relaciones muy valiosas con cursantes y docentes. (Víctor Penchaszadeh, Argentina).

\section{Lecciones aprendidas}

El curso Uisp en El Salvador marca la vida de las personas que participan en él, porque en el curso conocen verdaderamente la realidad: las raíces explicativas que les dan los elementos necesarios para juzgar su propia conducta, para entender la lucha de clases y para entender también porque la gente actúa como lo hacen y finalmente para entender la vida. El curso forma por ello activistas para toda la vida, para profundizar la lucha por la salud basada en la determinación social y por tanto para defender la Reforma de Salud.

El curso Uisp aborda la medicina social, las luchas en otros países y los diferentes procesos que se llevan a cabo para introducirlos en la comprensión del nuevo enfoque de la Determinación Social de Salud. El curso Uisp también da aportes 
significativos al pensamiento crítico. Los conocimientos aprendidos en el curso generan nuevas formas de trabajar en coordinación y solidaridad entre las personas. Les proporciona la posibilidad de creer, de imaginar, de soñar y de transformar la realidad a pesar de la crisis existencial, especialmente de la juventud.

Algunas personas afirman que su militancia ha crecido después del curso Uisp. Forman lazos de amistad entre las personas de las diferentes generaciones, y estos lazos inciden en su trabajo en salud, inclusive en el trabajo político. "Las redes se extienden y uno se siente parte de un colectivo", como dice unx de lxs excursillistas.

El curso Uisp ha permitido conocer cómo la alianza entre el imperialismo y la oligarquía se beneficia de la comercialización de la salud, utilizando estrategias para debilitar todo lo público con el fin de hacer de la salud un negocio.

El Curso Uisp me ha permitido aprender y desaprender, para reorientar mi camino hacia el sur y no hacia el norte como pensaba que era lo correcto. Aprendí que si quiero generar un impacto positivo en nuestra sociedad, debo empezar por hacer cambios en mi misma, comenzando desde mi forma de pensar y ver la realidad hasta la manera de hacer las cosas y luego involucrar a mi familia en ese proceso de cambio. (Excursillista).

El liderazgo del FNS menciona que el curso les desarrolla la capacidad de incidir en temas que parecieran que no son de salud, como el salario mínimo, el problema fiscal, las pensiones etc. Son asuntos que tienen que ver con salud y por lo tanto se debe participar e incidir en ellos. A partir del mismo conocimiento que se va adquiriendo, las acciones se van encaminando con enfoques más integrales y con una mayor claridad. Por lo tanto, es indispensable intervenir.

El programa del curso Uisp fortalece al FNS. Permite un cambio en la percepción del liderazgo del FNS y del personal de salud. Asegura la expansión territorial del FNS, procurando una mayor colaboración entre el liderazgo del FNS y el personal de Minsal que ha participado en el curso Uisp. (Miembro del FNS).

\section{Conclusiones}

El curso Uisp ha formado una masa crítica de trabajadorxs de la salud y de liderazgo del FNS sensibilizados y con herramientas para defender la Reforma de Salud. Lxs excursillistas son un grupo estratégico de relevo generacional en la salud pública, muchxs de ellxs están en puestos de trabajo de nivel gerencial en las instituciones y organizaciones a las que pertenecen. Realizar la Uisp en un país y con la participación de la mayoría de participantes procedentes del mismo, permite fortalecer la organización territorial.

El mayor obstáculo para el desarrollo de los cursos fue el profundo arraigo del modelo biomédico en la mayoría de profesionales de la salud, atribuible a su educación formal y a la organización y funcionamiento tradicional del sistema de salud. Otro obstáculo es la falta de seguimiento, respaldo institucional y financiamiento a los proyectos planteados en el curso Uisp.

La participación en los cursos Uisp en El Salvador ha permitido una transformación en términos ideológicos, teóricos y prácticos. No es simplemente una cuestión de semántica, sino entendimiento del enfoque de Determinación Social de la Salud. Una nueva concepción para abordar y entender el proceso salud-enfermedad. Un logro importante y una lección para el MSP a nivel mundial es el involucramiento de la masa crítica de cursillistas en este debate, así como su trascendencia a nivel nacional, regional y global.

La experiencia de El Salvador en la Reforma de Salud y el aporte de los cursos Uisp en este proceso, se resume en un documento publicado por el Minsal y titulado 'Diagnóstico Nacional de Promoción de la Salud'5, preparado conjuntamente por representantes del FNS y del Minsal. Este documento es un logro en sí mismo, es de importancia simbólica histórica.

La alianza estratégica generada entre el MSPUisp y el Minsal de El Salvador es de vital importancia. Por un lado, el MSP-Uisp pone a disposición del ministerio una metodología docente y un grupo de facilitadores entrenados en una pedagogía para la liberación, mientras que el 
Minsal asume como prioridad la formación de cuadros político-técnicos como elemento central para hacer avanzar la Reforma de Salud. Hoy, puede decirse que la conducción del sistema de salud salvadoreño está a cargo de egresados del curso Uisp que hacen de la lucha por el derecho a la salud su objetivo fundamental. (Oscar Feo, Venezuela).

Es muy importante la 'transversalidad' en la procedencia de los participantes y especialmente, la participación del FNS. La convivencia de los docentes favorece el intercambio de opiniones, dada la procedencia de entornos sanitarios, en ocasiones muy dispares. (Txema Ostolaza, País Vasco).

El compañero Juan Luis Uríadel País Vasco también ha hecho la siguiente reflexión:

No hay reforma de salud que permanezca en el tiempo sino la defienden sus profesionales, sus trabajadores y la población organizada. Ahí precisamente inciden los temas tratados en el curso Uisp en El Salvador.

Estudiante del séptimo curso Uisp, 2017, deja su reflexión:

En el transcurso del curso mi compromiso social y humano se fortaleció y me pregunté: ¿Realmente estoy haciendo lo suficiente por defender la salud de mi gente? ¿Existe un compromiso real más allá de estar atrás de un escritorio y un canapé? iNo! No basta conformarse con lo que los de 'arriba' hagan o quieran hacer, debe existir una participación social en donde todas y todos estemos involucrados y vayamos caminando juntos por defender nuestros derechos, pero además generar el cumplimiento de nuestros deberes buscando una sociedad en equilibrio y consecuente con lo que se dice, piensa, hace y siente. Durante la vivencia en el curso Uisp realizado en La Palma, todo fluyó para renovar nuestra mente y espíritu de lucha, nos transformamos para ser mejores personas, más humanos movidos por el amor a la vida y justicia social.
Y así, ¿Cómo se garantiza que el curso Uisp es un proceso que no tiene marcha atrás? "Más que ex-cursillistas, somos militantes de la lucha por la salud”. Varias personas opinan que la sostenibilidad de los cursos Uisp en El Salvador, no depende sólo de que el gobierno del FMLN o la visión actual de las autoridades del Minsal lo continúen o no. Garantizar la Uisp depende de la disposición de todos y todas, del seguimiento de lxs participantes así como de los cursos, los proyectos y los lazos de compañerismo y solidaridad ya desarrollados. También es relevante el apoyo de la cooperación internacional con una visión que fortalezca la Reforma de Salud a través de la formación de militantes. Todo esto requiere una reflexión para generar propuestas concretas en el tema de sostenibilidad.

El Dr. Eduardo Espinoza, ex viceministro de Políticas de Salud de Minsal y del equipo de coordinación de los cursos con la autora de este artículo opina y nos lleva hacia la conclusión.

Los cursos de la Universidad Internacional para la Salud de los Pueblos, cursos de Políticas de Salud para la Promoción y Abogacía del Derecho Humano a la Salud, o simplemente los 'cursos Uisp de La Palma', como fueron más popularmente conocidos, se constituyeron en un elemento omnipresente en el proceso de Reforma. Un crisol de formación de cuadros políticos comprometidos con el derecho a la salud de la población y un punto de referencia y debate entre el liderazgo comunitario, personal de salud y algunas de los más destacados pensadores y militantes por el derecho a la salud provenientes de muchos países, pero con fuerte predominancia latinoamericana. El 'Curso de La Palma' era esperado año con año y lo seguirá siendo. Quienes nos comprometimos con su diseño y realización no nos resignamos a que se interrumpan, son un elemento vital, renovador y energizante que no puede ni debe desaparecer.

Nunca se nos cruzó por la mente ofertar o solicitar un monto para compensar el tiempo o los conocimientos invertidos durante el curso o 
cobrarles su participación a los alumnos, sencillamente porque todos éramos allí alumnos, todos aprendíamos y nos fortalecíamos en la importancia de intercambiar, de exponer para que nuestras ideas fueran desmenuzadas, criticadas y retroalimentadas, por quienes eran la razón de ser de las mismas. Cada curso fue una experiencia diferente, enriquecedora e irrepetible.

El futuro podrá ser un tanto más difícil pues ya no contaremos con la disponibilidad de algunos recursos que nos facilitaban materialmente la tarea. Tendremos que encontrar oídos receptivos y gestionar con eficacia los recursos que se nos confíen, pero estamos seguros que los encontraremos para seguir formando nuevos combatientes

\section{Referencias}

1. El Salvador. Ministerio de Salud. Dirección de Legislación y Regulación en Salud. Viceministerio de Políticas de Salud. Construyendo la Esperanza: Estrategias y recomendaciones en salud 2009-2014. San Salvador: Minsal; 2010.

2. El Salvador. Ministerio de Salud. Dirección de Legislación y Regulación en Salud. Viceministerio de Políticas de Salud. Política Nacional de Salud 2015 -2019. San Salvador: Minsal; 2010.

3. Zúniga MH. Universidad Internacional para la Salud de los Pueblos El Salvador: Una experiencia transformadora. In: PHM. Case Studies in the IDRC Project 'Civil societyengagementforHealthforAll'. El Salvador: People'sHealthMovement; 2017. por el derecho a la salud que defiendan los logros alcanzados y que repliquen el entusiasmo y el crecimiento ideológico-político que dejan una huella especial en quienes como alumnos, como docentes, como facilitadores o como expertos en algún tema, se involucran en uno de estos cursos. Les esperamos como siempre, en La Palma, Chalatenango, en el próximo Curso Uisp.

\section{Colaboradora}

Zúniga MEH (0000-0002-6981-0170)* es responsable de redactar el manuscrito.
4. Zúniga MH, Espinoza E, Elías MA, et al. Sistematización de la experiencia de desarrollo de los cursos de la Universidad Internacional para la Salud de los Pueblos con el Ministerio de Salud de El Salvador en el periodo 2011 - 2017. San Salvador: Viceministerio de Políticas de Salud; Minsal; 2018.

5. El Salvador. Ministerio de Salud. Unidad de Promoción de la Salud, Viceministerio de Políticas de Salud.Diagnóstico Nacional de Promoción de la Salud 1945-2015. San Salvador: Minsal; 2015.

\footnotetext{
Recibido el 06/05/2019

Aprobado el 08/12/2019

Conflicto de intereses: inexistente
}

Apoyo financiero: no hubo 